\title{
Weiterer Beitrag zur Frage nach der Verwertung von tief abgebautem Eiweiß im tierischen Organismus.
}

\author{
VIII. Mitteilung. \\ Von \\ Emil Abderhalden.
}

(Aus dem physiologischen Institute der tierärztlichen Hochschule, Berlin.)

(Der Redaktion zugegangen am 26. August 1908.)

Durch eine Reihe von Versuchen darf es jetzt als unzweifelhaft festgestellt gelten, daß der tierische Organismus und speziell der Hund während langer Zeit seinen Eiweißbedarf mit vollständig abgebauten Proteinen decken kann. Wir haben noch mehrere Untersuchungen nach dieser Richtung ausgeführt. Das Resultat war stets eindeutig, sobald es gelang, das Versuchstier während längerer Zeit zur Aufnahme des Futters zu bewegen. Die verschiedenen Hunde zeigten ein sehr verschiedenes Verhalten. Manche beantworteten die Aufnahme des abgebauten Proteins bald mit Erbrechen, während andere das gleiche Präparat stets gerne aufnahmen und nie Widerwillen oder gar Erbrechen zeigten. Es sei im folgenden ein Versuch angeführt, bei dem das Nahrungseiweiß $\beta$ vollständig durch tief abgebautes Fleisch ersetzt worden war. Er dauerte vom 4. Juni bis zum 8. August, somit 36 Tage. Er wurde aus äußeren Gründen abgebrochen. Das Tier war stets munter und behielt sein Gewicht bei. Der Versuch wurde in genau derselben Weise durchgeführt, wie alle früher mitgeteilten, und ebenso war das abgebaute Fleisch in ganz entsprechender Weise gewonnen und auf den Grad seines Abbaus geprüft worden, wie wir es in den früheren Mitteilungen wiederholt geschildert haben. Bei der Durchführung dieses Versuches erfreute ich mich der Unterstützung des Herrn Sokolowski. 
Über Verwertung von tief abgebautem Eiweiß im Organismus. VIII. 349

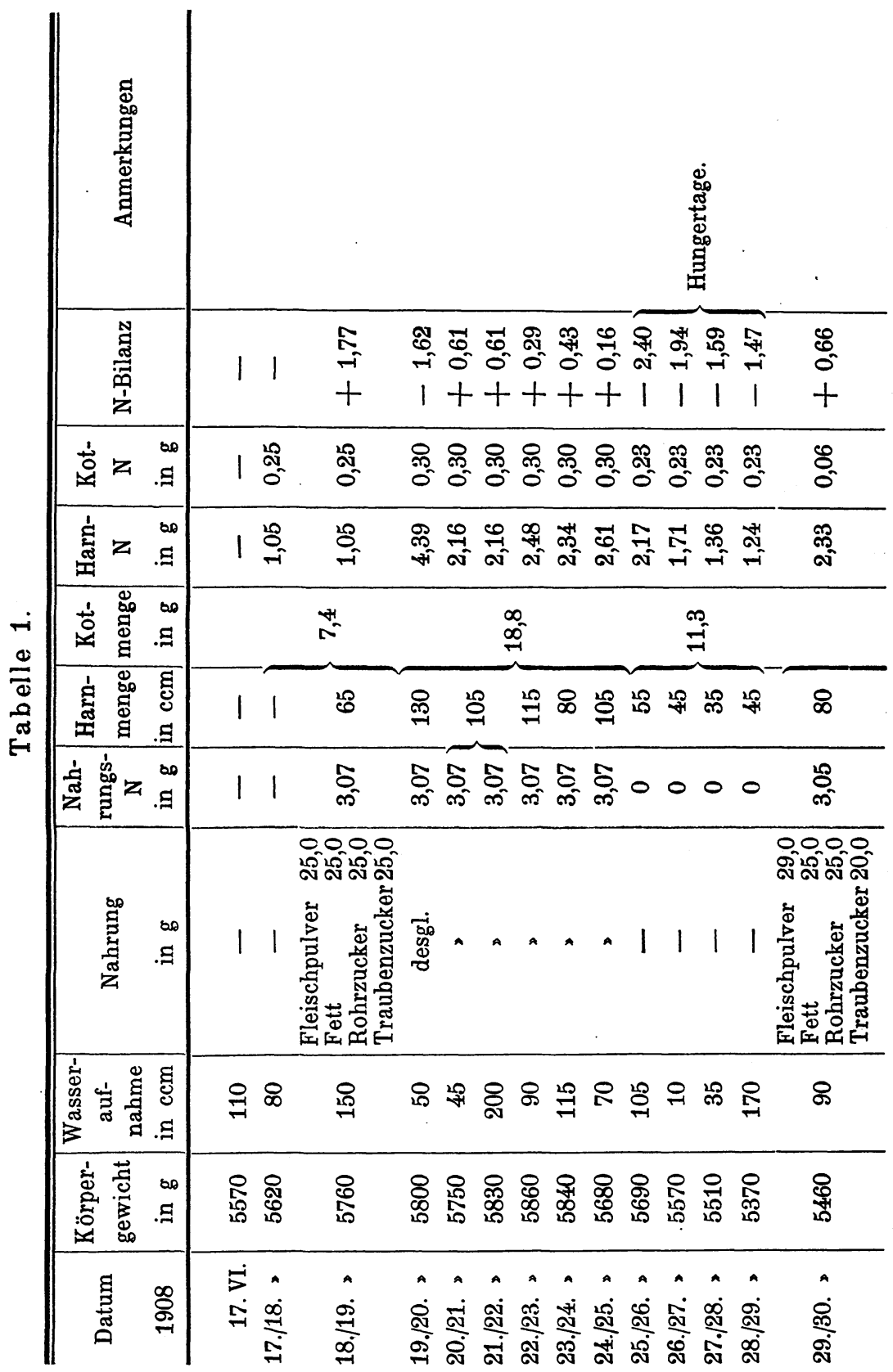

Hoppe-Seyler's Zeitschrift f. physiol. Chemie. LVII. 


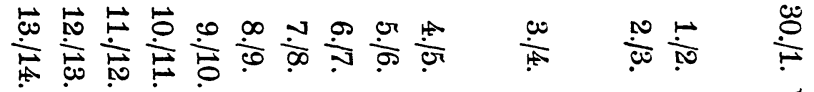

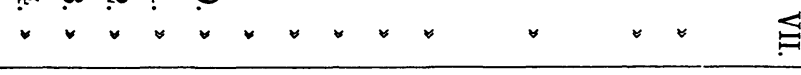

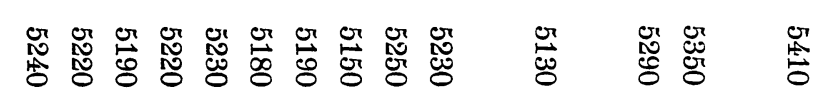

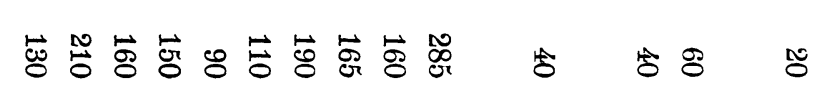

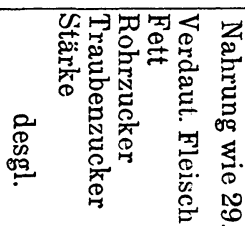
Nㅓ용

\section{—_}

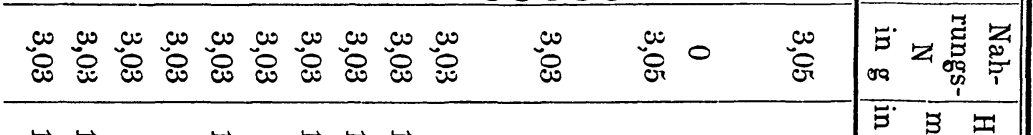

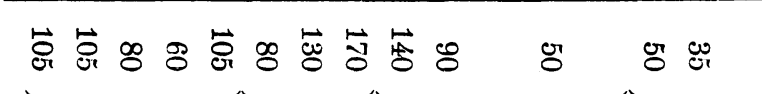

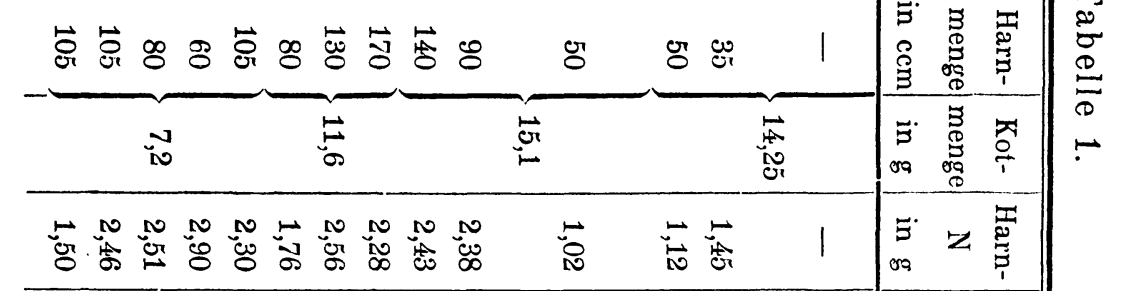

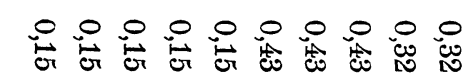

(1)

$+++1+t+t+t+1$

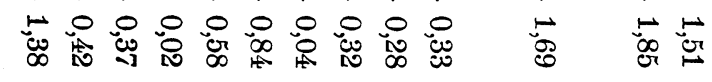


Über Verwertung von tief abgebautem Eiweiß im Organismus. VIII. 351

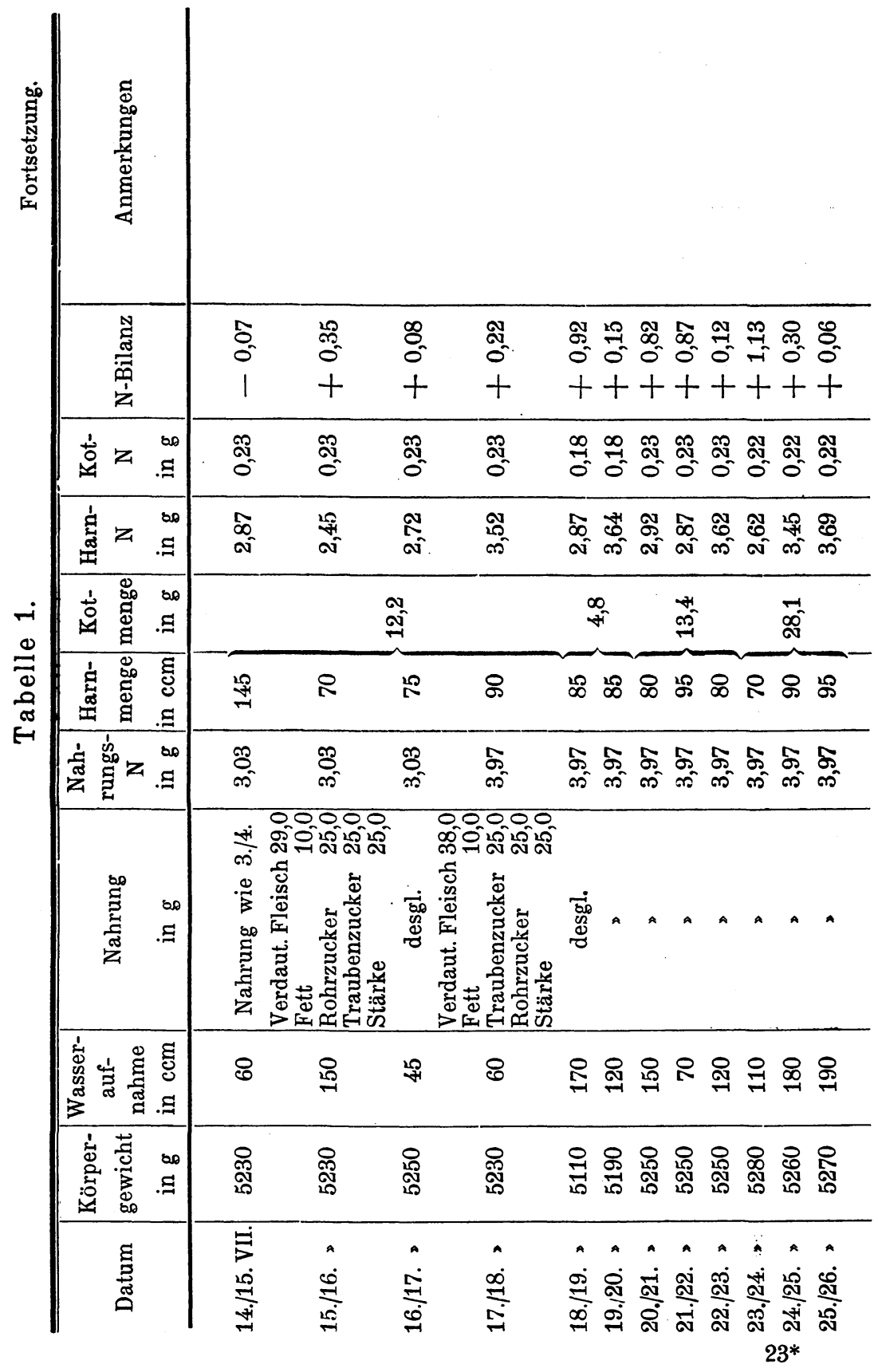




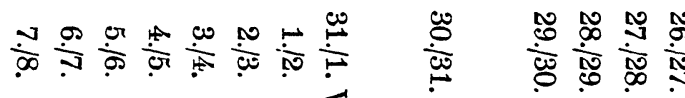

*.... .

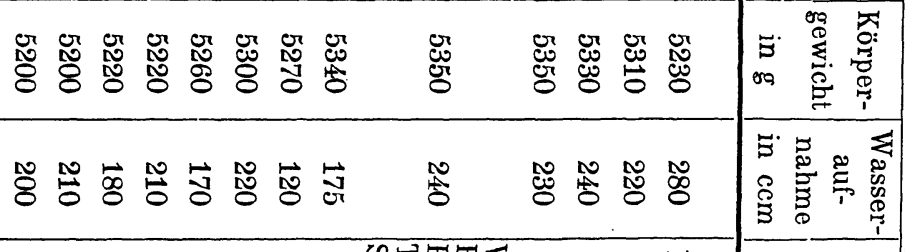

\begin{tabular}{|c|c|c|c|}
\hline 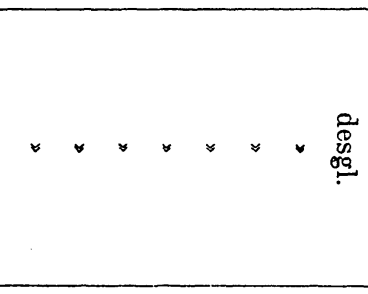 & 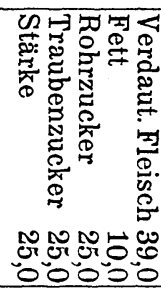 & 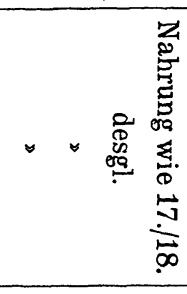 & 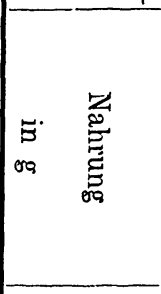 \\
\hline 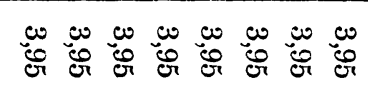 & $\underset{c}{w}$ & 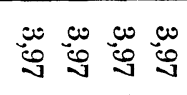 & 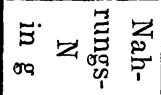 \\
\hline 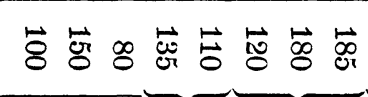 & $\overrightarrow{y ్ g}$ & 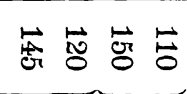 & 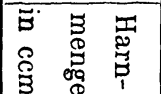 \\
\hline 䏒 & $\underset{\mathbb{C}}{\mathbb{N}}$ & $\stackrel{\infty}{\infty}$ & 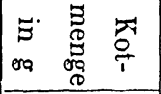 \\
\hline 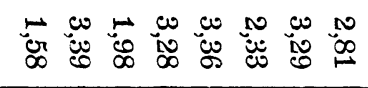 & $\stackrel{\infty}{\circ}$ & 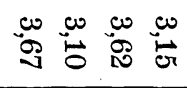 & 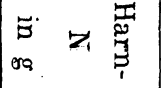 \\
\hline$|1| 1 \mid \stackrel{\circ}{\circ} \stackrel{0}{0} \stackrel{0}{0}$ & $\underset{\infty}{\infty}$ & 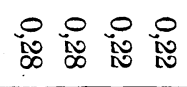 & 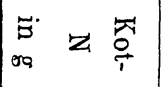 \\
\hline 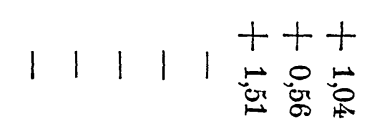 & $\begin{array}{l}+ \\
+ \\
\stackrel{0}{\circ}\end{array}$ & 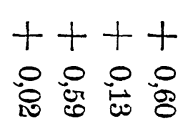 & 总 \\
\hline 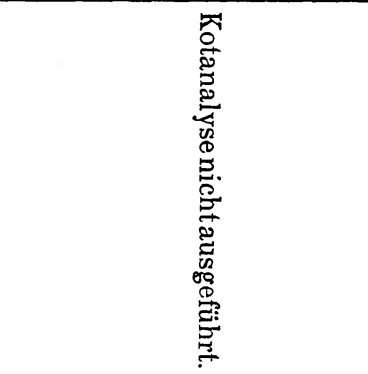 & 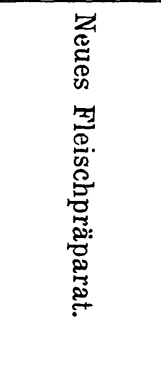 & & 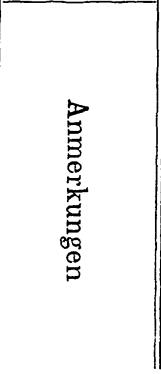 \\
\hline
\end{tabular}


Ein weiterer Versuch ist an einer trächtigen Hündin ausgeführt worden. Es war ausserordentlich schwer, dieses Versuchstier, von dessen Zustand wir zunächst nicht unterrichtet waren, ins Stickstoffgleichgewicht zu bringen. Es warf während des Versuches drei wohlentwickelte Junge. Wir haben den Versuch während der ersten Zeit der Lactation fortgeführt. Das Tier brach leider von Zeit zu Zeit jeweilen etwas von der eingegebenen Nahrung aus. Das Erbrochene wurde jedesmal eingedampft und wieder verfüttert. Der Versuch ist somit nicht so glatt ausgefallen, wie dies bei den früher mitgeteilten Untersuchungen der Fall war. Immerhin zeigt er ganz eindeutig, daß das abgebaute Fleisch, auch während der Lactation wenigstens für einige Zeit - den Eiweißbedarf des Versuchstieres vollständig deckte. Wir zweifeln nicht daran, daß es unter günstigeren Bedingungen gelingen würde, den Versuch über eine viel längere Zeit auszudehnen. Bei diesem Versuch erfreute ich mich der Mithilfe des Herrn Dr. Mètte. (Siehe Tabelle 2.)

Wir haben weiterhin auf Grund der bisherigen Erfahrungen über die Verwertung von tief abgebautem Eiweiß das ganze Problem nach anderer Richtung verfolgt. Einmal verwendeten wir Fleisch, das durch Säurehydrolyse vollständig aufgespalten worden war. Es ist uns geglückt, auch mit einem solchen Präparate Stickstoffgleichgewicht zu erhalten. Leider störten bald eintretende Nahrungsverweigerung und Erbrechen die Versuche. Wir setzen sie fort. Wir suchten ferner festzustellen, $\mathrm{ob}$ in dem Gemisch von Aminosäuren die eine oder andere Aminosäure fehlen kann. Zunächst gingen wir von vollständig abgebautem, $2^{1 / 2}$ Jahre lang verdautem Casein aus. Wir engten die Verdauungsflüssigkeit unter vermindertem Druck so lange ein, bis Krystallisation eintrat. Die Krystalle wurden abgesaugt. Sie waren natürlich nicht rein, sondern schlossen, wie die Untersuchung ergab, ganz erhebliche Mengen von Mutterlauge ein. Jedenfalls enthielt diese Fraktion alle in Casein vorhandenen Aminosäuren, dagegen überwogen die schwerlöslichen, wie Tyrosin und Leucin, ganz bedeutend. Die Mutterlauge dieser Krystallfraktion enthielt ebenfalls alle Aminosäuren, jedoch im 


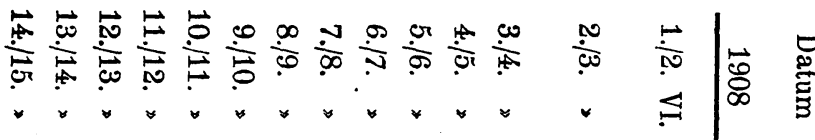

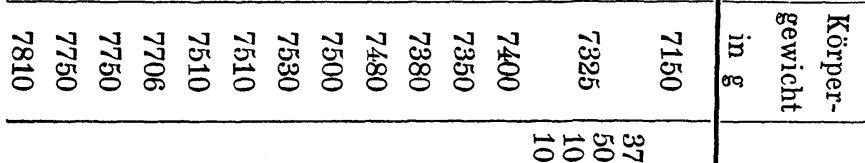

\begin{tabular}{|c|c|c|c|c|}
\hline 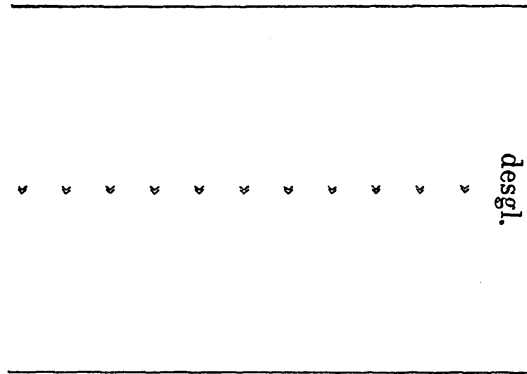 & 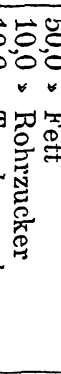 & 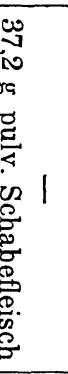 & \multirow{2}{*}{\multicolumn{2}{|c|}{ 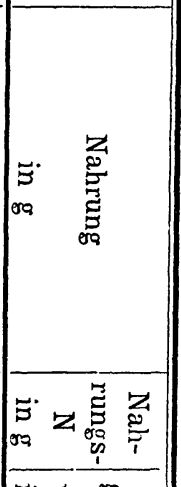 }} \\
\hline 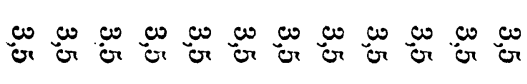 & $\underset{\sigma}{w}$ & & & \\
\hline 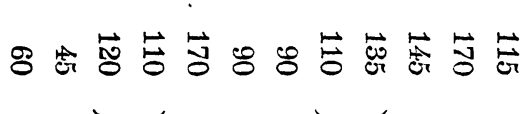 & \& & 1 & 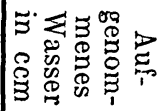 & \\
\hline \& $8 \overrightarrow{8} \&$ \& 点 & نे & 1 & 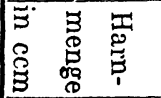 & \\
\hline 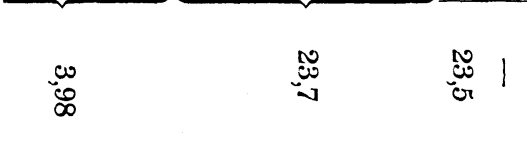 & 1 & 1 & 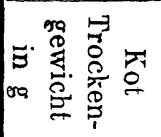 & \\
\hline 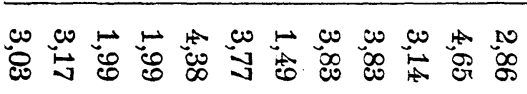 & iv & I & 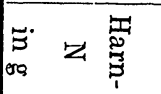 & \\
\hline 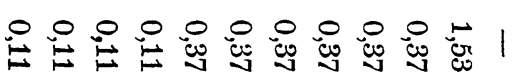 & 1 & 1 & $\underset{80}{F}$ 必 & \\
\hline 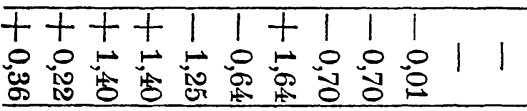 & 1 & 1 & 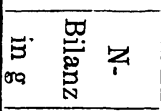 & \\
\hline & & 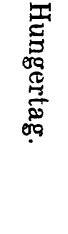 & 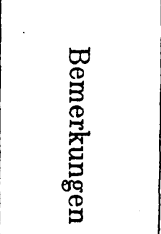 & \\
\hline
\end{tabular}


Über Verwertung von tief abgebautem Eiweiß im Organismus. VIII. 355

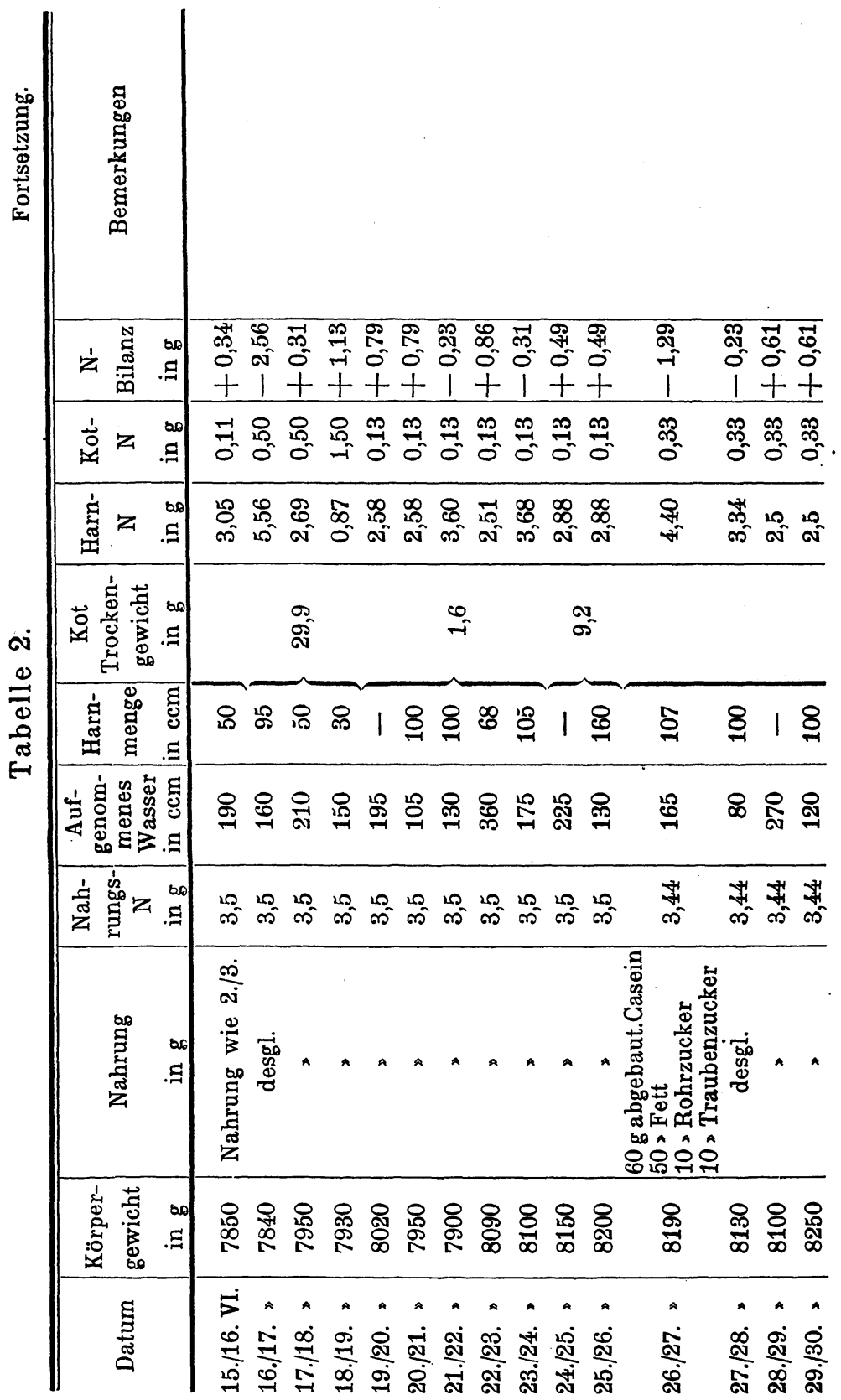




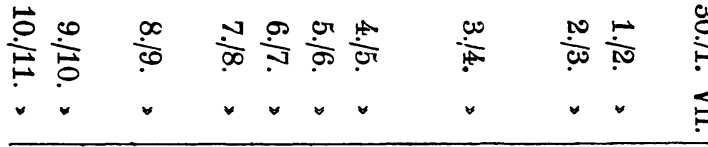

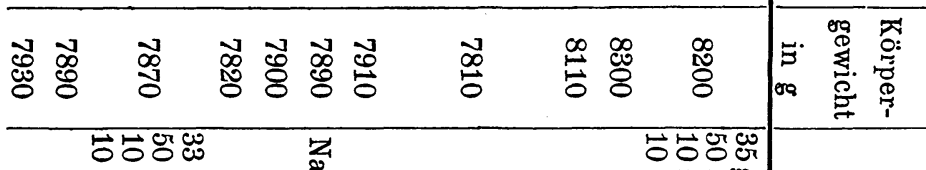

$\because \forall$ eq

究

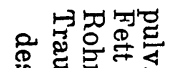

क्ष है:

究实念

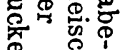

$\stackrel{\oplus}{\risingdotseq}$

눙엉

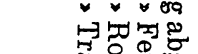

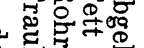

-

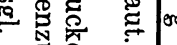

突蛋

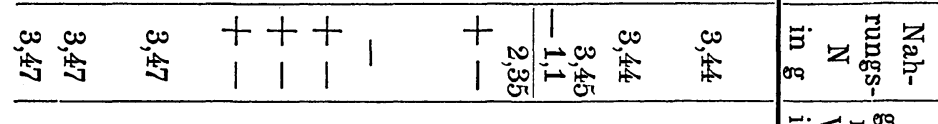

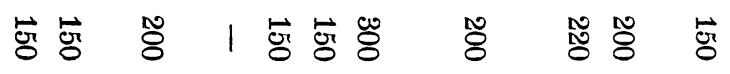

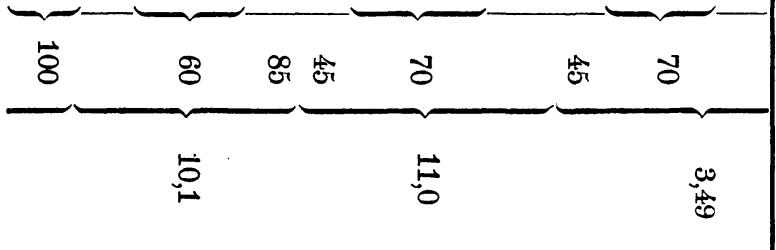

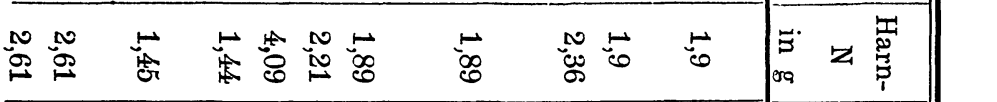

临

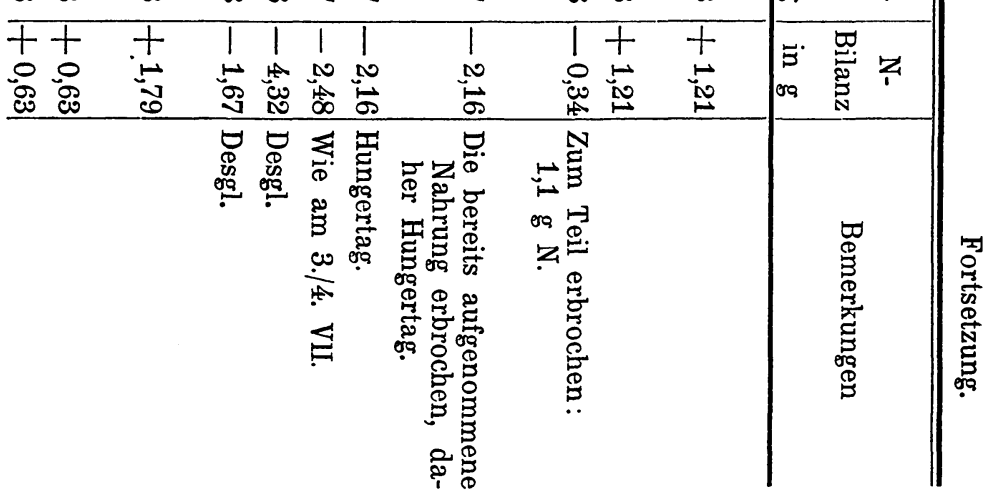


Über Verwertung von tief abgebautem Eiweiß im Organismus. VIII. 357

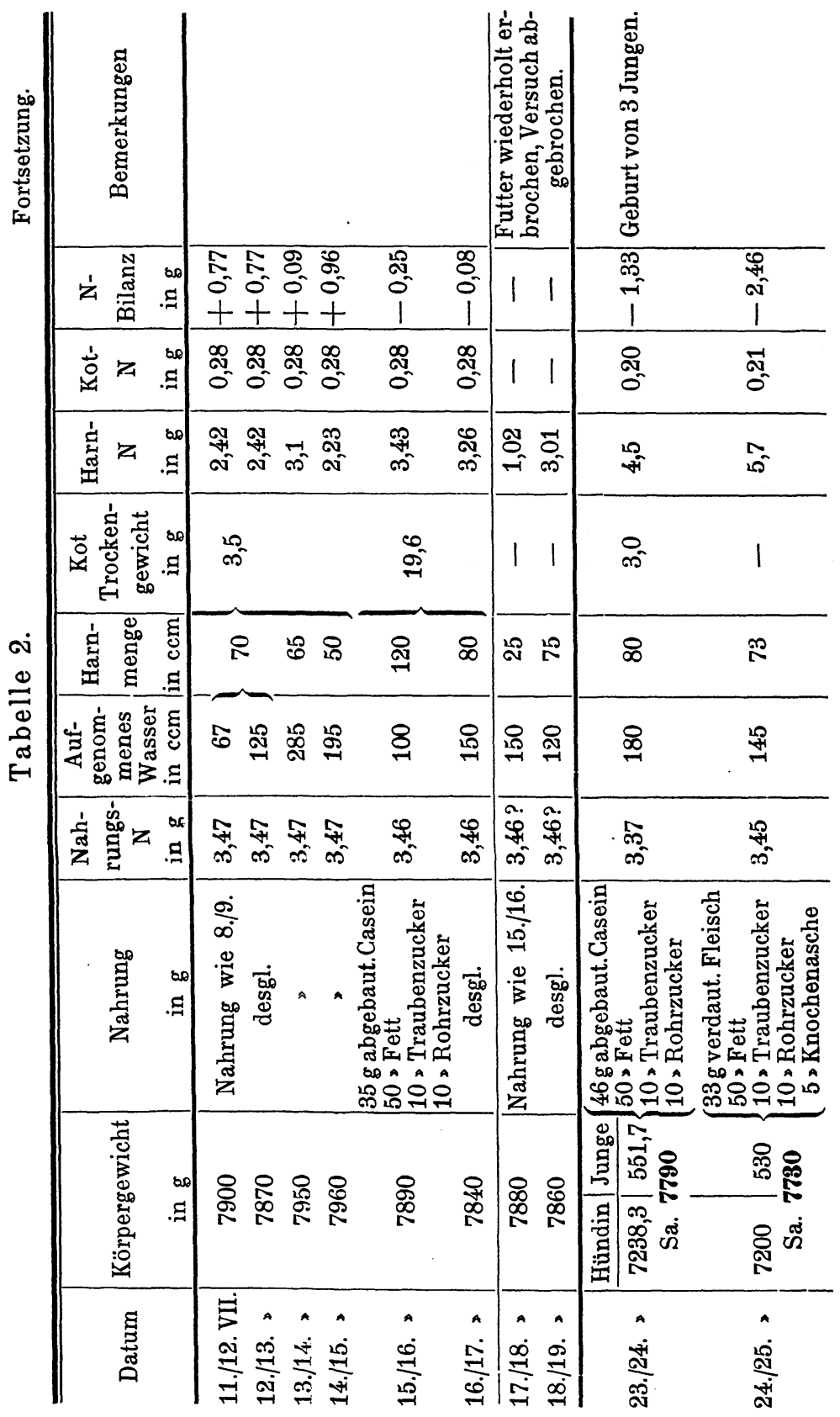


Emil Abderhalden,

\begin{tabular}{|c|c|c|c|c|c|c|c|}
\hline 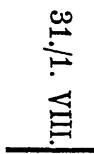 & 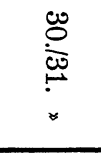 & ڤ. & 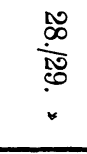 & $\frac{\sqrt{10}}{9}$ & 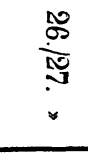 & $\begin{array}{c}\stackrel{0}{心} \\
\stackrel{\bar{N}}{\mathscr{D}} \\
\vdots \\
\vdots\end{array}$ & 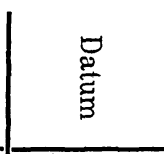 \\
\hline 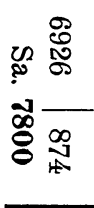 & 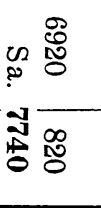 & 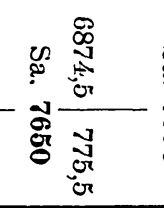 & 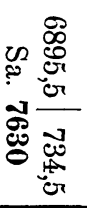 & 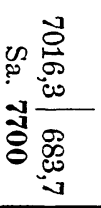 & 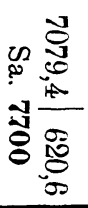 & 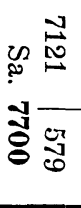 & 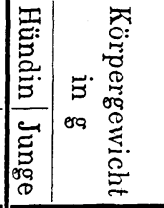 \\
\hline 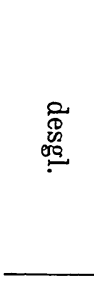 & 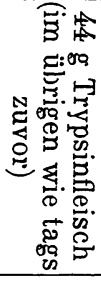 & 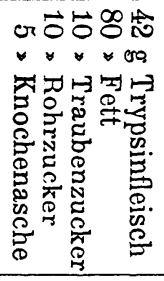 & 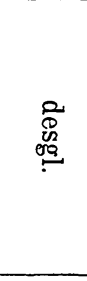 & 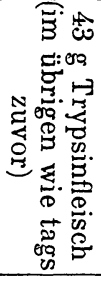 & 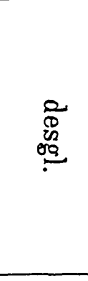 & 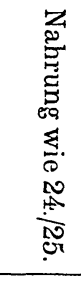 & 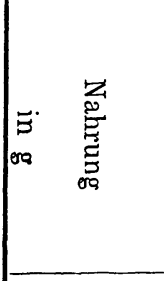 \\
\hline 点 & 整 & 숭 & 䓯 & 占 & 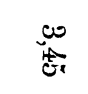 & 禹 & 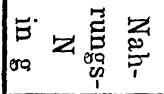 \\
\hline 总 & $\vec{\circ}$ & $\overrightarrow{\tilde{g}^{\prime}}$ & $\stackrel{N}{0}$ & 焉 & $\tilde{8}$ & $\stackrel{\rightleftarrows}{\sigma}$ & 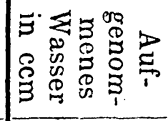 \\
\hline 8 & $\infty$ & 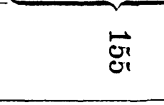 & & & \& & & 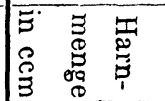 \\
\hline $\begin{array}{l}\infty \\
\infty \\
\infty\end{array}$ & $\begin{array}{c}\stackrel{1}{N 0} \\
-\infty\end{array}$ & $\begin{array}{c}\vec{\omega} \\
\infty \\
\infty\end{array}$ & 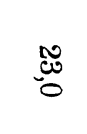 & & $\begin{array}{l}\text { N } \\
\text { N } \\
\text { O }\end{array}$ & & 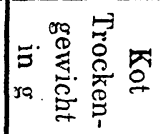 \\
\hline 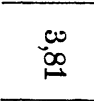 & W్ & w & 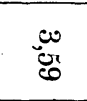 & 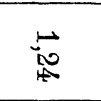 & 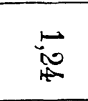 & 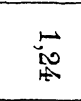 & 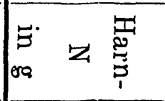 \\
\hline ○్ల్ర & 隹 & 尽 & $\stackrel{0}{\omega ు}$ & $\underset{10}{0}$ & 足 & 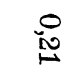 & 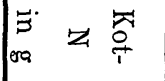 \\
\hline $\begin{array}{l}+ \\
0 \\
0 \\
0\end{array}$ & $\begin{array}{l}+ \\
0 \\
0 \\
\text { o } \\
\end{array}$ & $\begin{array}{l}+ \\
0 \\
0 \\
0 \\
1\end{array}$ & $\begin{array}{l}+ \\
\vdots \\
\vdots \\
\vdots\end{array}$ & $\begin{array}{c}+ \\
\cdots \\
0 \\
0 \\
c\end{array}$ & $\begin{array}{l}+ \\
1 \\
8\end{array}$ & $\begin{array}{l}+ \\
1 \\
8 \\
\end{array}$ & 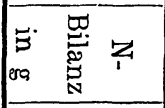 \\
\hline & & & & & & & 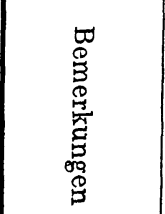 \\
\hline
\end{tabular}


w.esentlichen nur die leichtlöslichen. Es fehlte somit keiner Fraktion eine Aminosäure vollständig. Es war nur das Mengenverhältnis, in dem die einzelnen Bausteine vorhanden waren, ein verschiedenes. Wir fütterten nun einen Terrier einmal mit dem Gemisch beider Fraktionen, dann mit der Fraktion I und schlieBlich mit der Mutterlauge (Fraktion II) der Krystallfraktion (I). Wir haben absichtlich dem Versuchstiere mehr Stickstoff zugeführt, als zur Erhaltung des Stickstoffgleichgewichts notwendig war. Bei Verfütterung des Gemisches von Fraktion I und II trat, wie die folgende Tabelle zeigt, bald Stickstoffretention ein. In den letzten drei Tagen retinierte das Versuchstier durchschnittlich 1,26 g Stickstoff. Nach Eingabe der Fraktion I trat sofort eine Verschlechterung der Stickstoffbilanz ein. Das gleiche war der Fall, als die Mutterlauge der Krystallfraktion die ausschließliche Stickstoffzufuhr bildete. Leider konnten wir nicht, wie wir es gewünscht hatten, einen Kontrollversuch mit einer Mischung der Fraktion I und II anschließen, um dem Einwand zu begegnen, daß die größtenteils negative Stickstoffbilanz auf die lange Dauer des Versuches zurückzuführen sei. Dieser Einwand ist nach unseren sonstigen Erfahrnngen kaum begründet. Der Ausfall des ganzen Versuches scheint dafür zu sprechen, daß die Verwertung der Bestandteile eines Gemisches von Eiweißabbauprodukten durch den tierischen Organismus sich nach dem Gesetz des Minimums richtet. Die Stickstoffzufuhr war in allen Fällen dieselbe und ebenso waren in allen drei Versuchsabschnitten alle Aminosäuren zugegen. Ihre Verteilung war nur eine verschiedene und das kommt auch in der Stickstoffbilanz deutlich zum Ausdruck. Dieser, gemeinschaftlich mit Herrn Sokolowski durchgeführte Versuch zeigt gleichzeitig, wie notwendig es ist, bei derartigen Untersuchungen möglichst vom Stickstoffminimum auszugehen. Wir werden auch diesen Versuch unter anderen Bedingungen wiederholen. (Siehe Tabelle 3.)

Endlich haben wir aus einem Verdauungsgemisch von Casein das Tryptophan seiner Hauptmenge nach entfernt, indem wir es in der bekannten Weise mit Quecksilbersulfat in schwefelsaurer Lösung fällten. Aus dem Filtrat des Niederschlages 


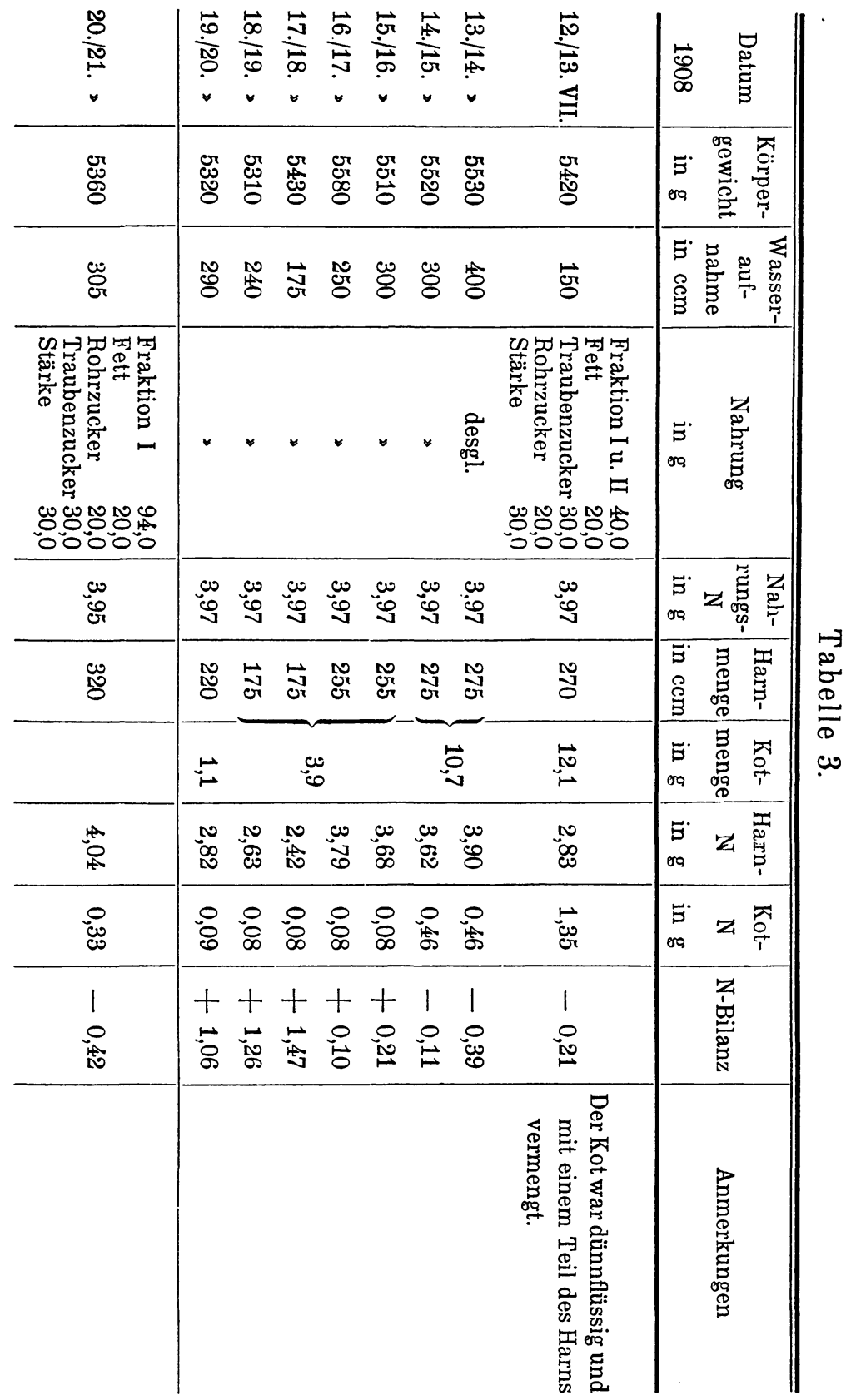


Über Verwertung von tief abgebautem Eiweiß im Organismus. VIII. 361

\begin{tabular}{|c|c|c|c|}
\hline 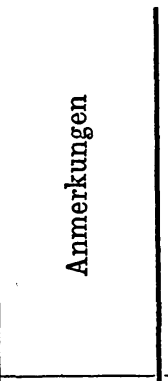 & 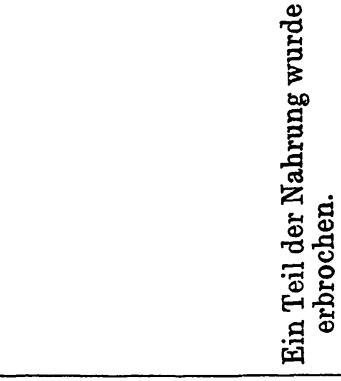 & & \\
\hline 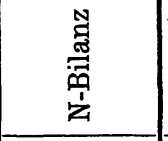 & 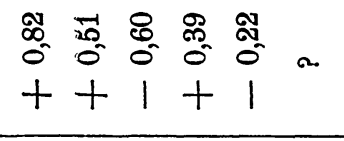 & $\begin{array}{l}5 \\
0 \\
0 \\
1\end{array}$ & $\begin{array}{lll}\text { से } & \text { F⿱ } \\
0 & 0 \\
1 & 1\end{array}$ \\
\hline $\begin{array}{lll}\dot{0} & \infty & \infty \\
0 & \Xi\end{array}$ & 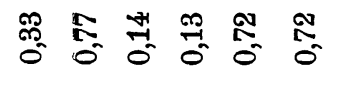 & 亲 & 양 양 1 \\
\hline 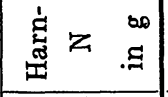 & 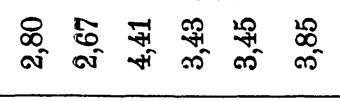 & क्ष & 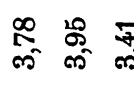 \\
\hline 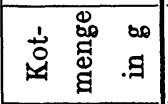 & 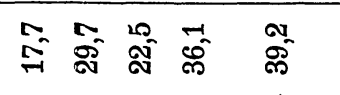 & ثै & 1 \\
\hline 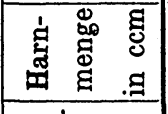 & 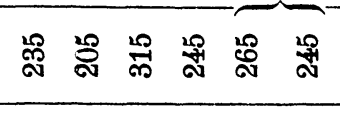 & 号 & ర్లి న్లి \\
\hline 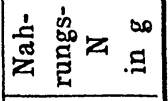 & 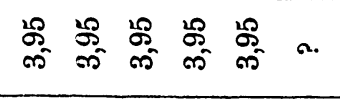 & $\underset{\infty}{\infty}$ & क् \\
\hline 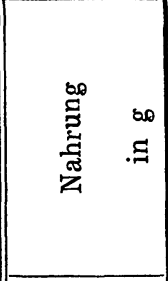 & 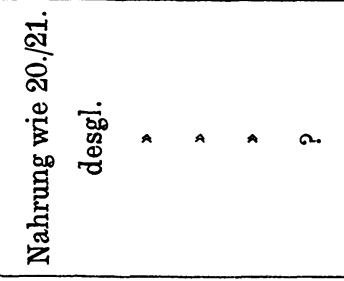 & 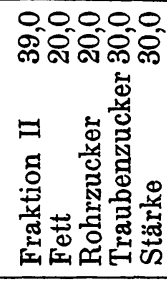 & 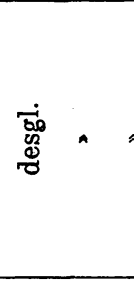 \\
\hline 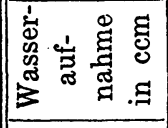 & ๙్సి 각 \& \& & 유 & 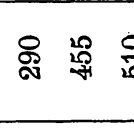 \\
\hline 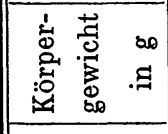 & 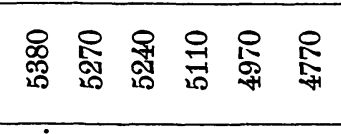 & ஓ্ণ & 윽 융 \\
\hline 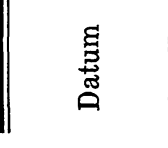 & 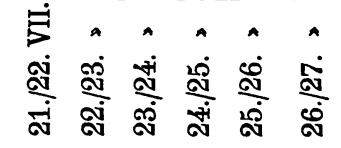 & $\frac{\hat{\infty}}{\stackrel{\leftrightarrow}{\grave{N}}}$ & 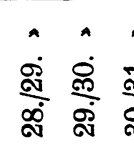 \\
\hline
\end{tabular}


Emil Abderhalden,

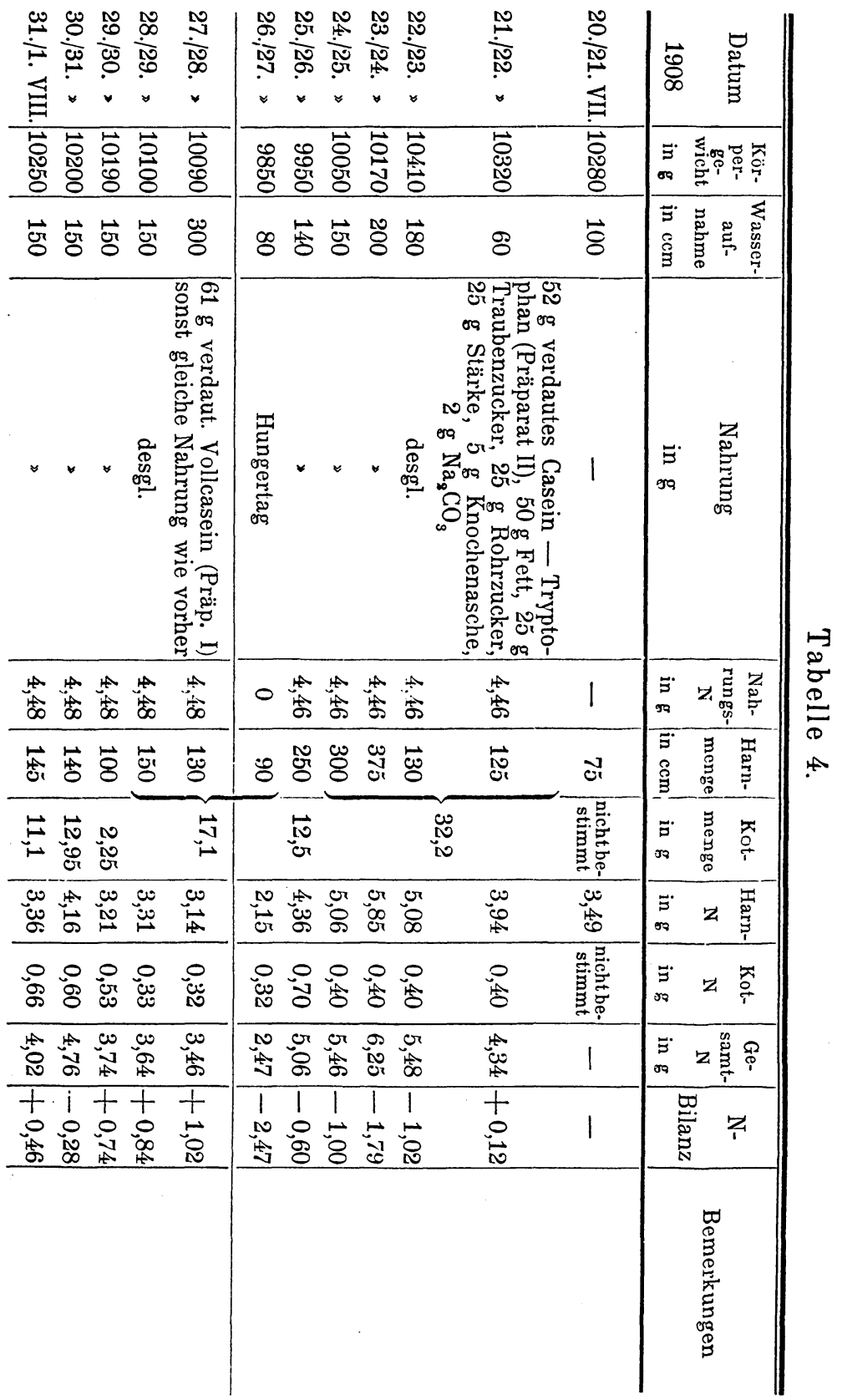


Über Verwertung von tief abgebautem Eiweiß im Organismus. VIII. 363

\begin{tabular}{|c|c|c|c|c|c|c|}
\hline 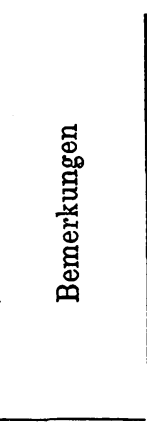 & & 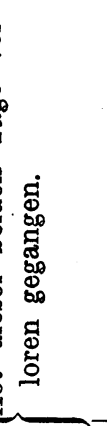 & 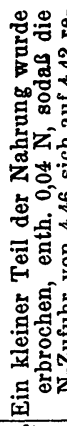 & & 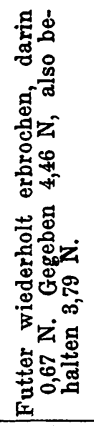 & 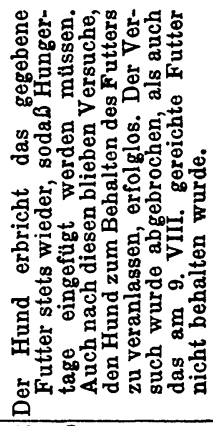 \\
\hline 学 䓴 & $\begin{array}{l}0 \\
0 \\
0 \\
+\end{array}$ & 1 & $\stackrel{0}{-1}$ & $\stackrel{\substack{1 \\
i}}{1}$ & \begin{tabular}{l}
$F$ \\
\multirow{1}{0}{} \\
1
\end{tabular} & $\begin{array}{ll}0 & 0 \\
\text { me } & 0 \\
\text { of } & \text { m } \\
1 & 1\end{array}$ \\
\hline 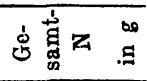 & $\stackrel{\infty}{\infty}_{\forall}^{\infty} 1$ & 1 & $\begin{array}{l}5 \\
4 \\
15\end{array}$ & $\frac{1}{82}$ & ๙ & 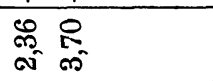 \\
\hline$\stackrel{\Delta}{4}$ z & $\begin{array}{ll}0 \\
0 \\
0\end{array}$ & 1 & ח̊ & $\begin{array}{c}\overrightarrow{8} \\
0 \\
0\end{array}$ & ח్ & 웅여 \\
\hline 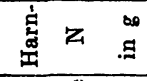 & 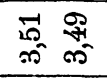 & F & $\frac{41}{25}$ & $\begin{array}{l}28 \\
10 \\
20\end{array}$ & $\begin{array}{l}\vec{\infty} \\
\infty\end{array}$ & क् \\
\hline 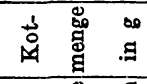 & जิ 1 & 1 & 1 & $\begin{array}{l}20 \\
\stackrel{10}{9}\end{array}$ & 1 & 1 点 \\
\hline 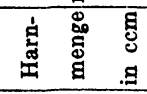 & ర్తి ๙ి & 品 & in & $\stackrel{20}{7}$ & \&্ণ & \& \\
\hline 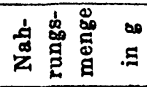 & 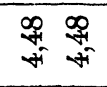 & $\mathscr{q}$ & $\underset{\text { If }}{*}$ & $\mathscr{L}_{41}$ & 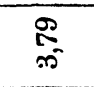 & 11 \\
\hline 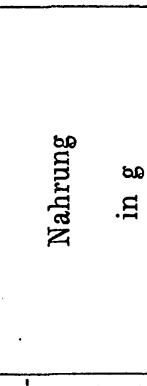 & 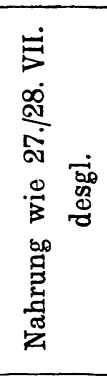 & 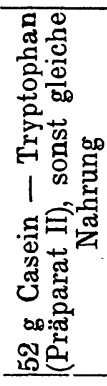 & $\begin{array}{l}\dot{80} \\
\dot{80} \\
\dot{0}\end{array}$ & 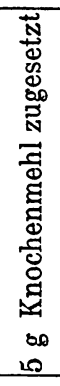 & 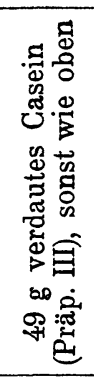 & 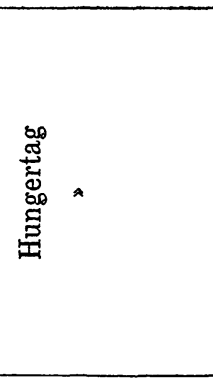 \\
\hline 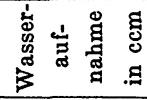 & 㽞 & $\stackrel{9}{-1}$ & $\$$ & 吕 & 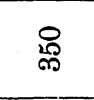 & 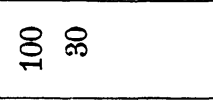 \\
\hline 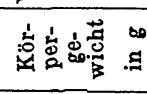 & 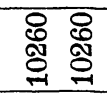 & ৪্ণ & 8 & $\begin{array}{l}\text { \& } \\
\& \\
\circ\end{array}$ & $\begin{array}{l}8 \\
8 \\
\infty \\
0\end{array}$ & 용 \&్రి \\
\hline 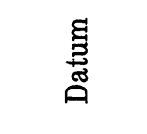 & 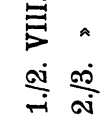 & $\frac{a}{a+1}$ & $\hat{\dot{\rho}} \dot{\vec{i}}$ & $\dot{0}$ & $\frac{\hat{r}}{6}$ & $\hat{\infty} \frac{\hat{0}}{\dot{\infty}}$ \\
\hline
\end{tabular}


entfernten wir die Schwefelsäure quantitativ mit Baryt und das Quecksilber mit Schwefelwasserstoff. Durch das Filtrat vom Quecksilbersulfid leiteten wir so lange Luft durch, bis der Geruch nach Schwefelwasserstoff verschwunden war. Es wurde dann unter vermindertem Drucke zur Trockene eingedampft. Der verbleibende Rückstand war nicht ganz tryptophanfrei. Jedenfalls war der größte Teil dieser Aminosäure entfernt worden. Auch vom Tyrosin war, wie die Untersuchung ergab, ein Teil mit ausgefällt worden.

Der Versuchsplan war nun folgender: Es sollte ein Hund mit tief abgebautem Casein (Präparat I) ins Stickstoffgleichgewicht gebracht werden. Dann wollten wir den gesamten Stickstoffbedarf durch das gleiche Präparat (II) ersetzen, dem jedoch die Hauptmenge des Tryptophans entzogen worden war. Zur Kontrolle sollte dann das gleiche Versuchstier schließlich das letztere Präparat + einem Zusatz von l-Tryptophan und l-Tyrosin erhalten (Präparat III). Leider ist es uns nicht geglückt, einen derartigen Versuch vollständig durchzuführen. In dem einen Versuche hatten wir mit demjenigen Präparate begonnen, dem Tryptophan und Tyrosin wieder zugesetzt worden waren. Das Versuchstier war 6 Tage im Stickstoffgleichgewicht. Beim Übergang zum Präparat II (- Tryptophan) brach das Versuchstier. Wir haben eine ganze Anzahl derartiger Versuche durchgeführt. Immer störte eintretendes Erbrechen die ganze Versuchsreihe. Der folgende unter Mitwirkung des Herrn Schrage durchgeführte $V$ ersuch ist der am besten gelungene. Er versagte leider beim Beginne der Nachperiode mit Präparat III. (Siehe Tabelle 4.)

Aus diesem Versuche geht ohne weiteres hervor, daß das des größten Teils seines Tryptophans und eines Teils seines Tyrosins beraubte Casein bei weitem nicht in gleichem Maße imstande war, Eiweiß zu ersetzen, wie das alle Bausteine enthaltende Verdauungsgemisch aus Casein. Wir setzen auch diese Versuche fort und hoffen, schließlich zu einer vollständigen Versuchsserie gelangen $\mathrm{zu}$ können. 